\title{
Role of molecular turnover in dynamic deformation of a three-dimensional cellular membrane
}

\author{
Satoru Okuda ${ }^{1,2} \cdot$ Mototsugu Eiraku $^{1}$
}

Received: 8 December 2016 / Accepted: 13 May 2017 / Published online: 29 May 2017

(C) The Author(s) 2017. This article is an open access publication

\begin{abstract}
In cells, the molecular constituents of membranes are dynamically turned over by transportation from one membrane to another. This molecular turnover causes the membrane to shrink or expand by sensing the stress state within the cell, changing its morphology. At present, little is known as to how this turnover regulates the dynamic deformation of cellular membranes. In this study, we propose a new physical model by which molecular turnover is coupled with three-dimensional membrane deformation to explore mechanosensing roles of turnover in cellular membrane deformations. In particular, as an example of microscopic machinery, based on a coarse-graining description, we suppose that molecular turnover depends on the local membrane strain. Using the proposed model, we demonstrate computational simulations of a single vesicle. The results show that molecular turnover adaptively facilitates vesicle deformation, owing to its stress dependence; while the vesicle drastically expands in the case with low bending rigidity, it shrinks in that with high bending rigidity. Moreover, localized active tension on the membrane causes cellular migration by driving the directional transport of molecules within the cell. These results illustrate the use of the pro-
\end{abstract}

Electronic supplementary material The online version of this article (doi:10.1007/s10237-017-0920-8) contains supplementary material, which is available to authorized users.

Satoru Okuda

okuda@cdb.riken.jp

1 Laboratory for in vitro Histogenesis, Center for Developmental Biology (CDB), RIKEN, 2-2-3 Minatojima-minamimachi, Chuo-ku, Kobe, Hyogo 650-0047, Japan

2 JST PRESTO, 4-1-8 Honcho, Kawaguchi, Saitama 332-0012, Japan posed model as well as the role of turnover in the dynamic deformations of cellular membranes.

Keywords Cytomembrane $\cdot$ Molecular turnover $\cdot$ Triangular mesh model $\cdot$ Mechanosensing $\cdot$ Coarse-grained modeling . Multiscale simulation

\section{Introduction}

Cellular membranes dynamically extend and shrink by removing and replacing their molecular components such as the constituents of their phospholipid and lining cytoskeletons (Staykova et al. 2011). For example, during the lamellipodia formation, the cytomembrane projects outwards as lipid molecules diffuse from within the surrounding membrane and are transported from the cytoplasm (Keren 2011). Moreover, in the process of epithelial mitosis, cells deform to become round as they decrease their surface area, with lipid molecules being transported into the cytoplasm (Raucher and Sheetz 1990). In these cases, membrane deformations are actively driven by forces generated within the cell, i.e., actomyosin contractile forces. Importantly, in general, the membrane morphology is geometrically constrained by its volume-area balance (Ghosh and Singh 1992). Owing to this constraint, deforming cellular membranes requires changing the number of membrane molecules. Thus, molecular turnover has a crucial role in regulating cellular membrane deformations.

This turnover can be regulated by membrane-associated proteins (Peters et al. 2016; McMahon and Gallop 2005; Kozlov et al. 2010). On a molecular scale, these proteins are collectively localized on the membrane and form complexes to pinch off parts of the membrane as liposomes. These liposomes are transported to another membrane and fused 
through the activities of membrane-associated proteins, such as SNAREs (Grant and Donaldson 2009). Notably, these proteins are known to play the role of mechanosensors, owing to the dependence of adhesion upon the membrane-stress state (Kozlov et al. 2010). Therefore, understanding the effects of turnover upon membrane deformation requires analyzing the feedback from the membrane-stress state on the turnover.

Several computational methods have been proposed to analyze the dynamics of cellular membranes. At a molecular level, molecular dynamics methods have been often used, whereby individual atoms are expressed as particles. Because the membrane dynamics are realistically expressed on the scale of individual lipid molecules, turnover in these methods physically results from molecular interactions (van der Ploeg and Berendsen 1982; Heller et al. 1993; Chiu et al. 1995). On the contrary, at a continuum level, several coarse-graining models have been proposed, ignoring the degrees of freedom of individual lipid molecules (Gompper and Kroll 1997; Ho and Baumgärtner 1990; Boal and Rao 1992; Gompper and Kroll 2004; Zhao and Kindt 2005). In particular, triangular mesh models have often been used to analyze the macroscopic dynamics of organelles and cytomembranes (Noguchi and Gompper 2005b; Ramakrishnan et al. 2013, 2015), and also applied to the analyses of cellular mechanotransduction (Atilgan and Sun 2007; Powers et al. 2012, 2014). In these models, membrane morphology is expressed by a triangular meshwork and membrane fluidity is successfully expressed by dynamically remodeling the meshwork topology (Gompper and Kroll 1998). Thus, modeling the membrane turnover on a triangular mesh model yields a powerful tool for investigating the dynamics of cellular membranes.

In this study, we propose a new computational model for simulating the turnover-dependent dynamics of threedimensional cellular membranes. Firstly, we propose topological operations on a triangular network to express the plastic extension and shrinkage of a membrane. Secondly, we propose stochastic descriptions of molecular transport that depend upon the membrane-stress state. Thirdly, using the proposed model, we demonstrate computational simulations of several membrane dynamics and explore the effects of turnover upon membrane deformation. Finally, we discuss the applicability of the proposed model and report new findings on the effects of turnover upon membrane deformations.

\section{Multiscale modeling of turnover-dependent membrane dynamics}

\subsection{Description of three-dimensional membrane deformation}

The membrane shape is expressed by a triangular meshwork (Fig. 1a), whereby the membrane surface is expressed by a patch of triangles (Fig. 1b, c).

The membrane dynamics are governed by an equation for the motion of vertices. By representing the position vector of the $i$ th vertex by $\boldsymbol{r}_{i}$, the vertex motion obeys the over-dumped Langevin equation as follows:

$\eta \frac{\mathrm{d} \boldsymbol{r}_{i}}{\mathrm{~d} t}=-\nabla U+\boldsymbol{w}_{i}$

The left-hand side of Eq. (1) indicates a frictional force exerted on the $i$ th vertex. Scalar $\eta$ is a friction coefficient between the membrane and its microenvironment. The righthand side of Eq. (1) denotes the energetic force acting on the $i$ th vertex, where $U$ is an effective energy function. Variable $\boldsymbol{w}_{i}$ is the Gaussian noise exerted on the $i$ th vertex that satisfies the following statistics:

$$
\begin{aligned}
& \left\langle\boldsymbol{w}_{i}(t)\right\rangle=\mathbf{0}, \\
& \left\langle\boldsymbol{w}_{i}(t) \otimes \boldsymbol{w}_{j}\left(t^{\prime}\right)\right\rangle=2 \eta k_{B} T \delta_{i j} \delta\left(t-t^{\prime}\right) \mathbf{1},
\end{aligned}
$$

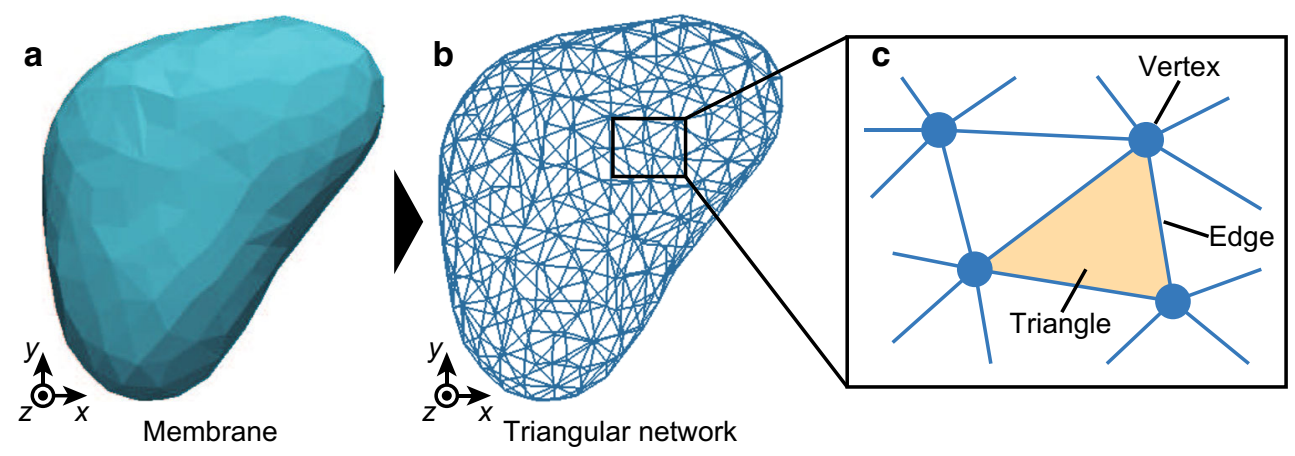

Fig. 1 Triangular membrane model. a Single membrane. b Triangular network composing the single membrane shown in a. c Schematic diagram of the triangular network. Membrane morphology is expressed by a patch of triangles, wherein individual triangles share their vertices and edges with their neighbors 
where $\langle\ldots\rangle$ is a statistical average, $\mathbf{0}$ is the zero vector, and $\mathbf{1}$ is the second-order unit tensor. Constant $k_{B}$ is the Boltzmann constant and $T$ is the effective temperature.

Effective energy $U$ is given by

$U=U_{\mathrm{cc}}+U_{\mathrm{eff}}+U_{\mathrm{act}}$,

where $U_{\text {cc }}, U_{\text {eff }}$, and $U_{\text {act }}$ are the constraint, effective, and active energy functions, respectively.

From a numerical viewpoint, to maintain a discrete size for the triangular mesh, we describe the constraint energy, $U_{\mathrm{cc}}$, by the following function:

$\left.U_{\mathrm{cc}}=\sum_{i}^{\text {vertex vertex }} \sum_{j(>i)}^{K_{\mathrm{r}}} \frac{l_{i j}}{2}-1\right)^{2} \delta_{\left[l_{i j}<l_{\mathrm{rep}}\right]}$,

where $\delta_{[\alpha]}$ is a binary function of the condition $\alpha$. Here, $K_{\mathrm{r}}$ and $l_{\text {rep }}$ are the repulsive modulus and distance, respectively.

Membrane mechanics are described by the effective energy, $U_{\text {eff }}$. Here, we introduce the $i$ th cell volume $v_{i}$, the $i$ th triangle area $a_{i}$, the mean curvature around the $i$ th vertex $M_{i}$ and the mean surface area around the $i$ th vertex $A_{i}$. Using these variables, $U_{\text {eff }}$ is simply described by

$$
\begin{aligned}
U_{\text {eff }}= & \sum_{i}^{\text {vesicle }} \frac{K_{\mathrm{v}}}{2}\left(\frac{v_{i}}{v_{\text {eq } i}}-1\right)^{2} \\
& +\sum_{i}^{\text {triangle }} \frac{K_{\mathrm{a}}}{2}\left(\frac{a_{i}}{a_{\mathrm{eq}}}-1\right)^{2} \\
& +\sum_{i}^{\text {vertex }} 2 K_{\mathrm{c}}\left(\frac{M_{i}^{2}}{A_{i}}\right) .
\end{aligned}
$$

The first term indicates the volume elastic energy of individual vesicles, where $K_{\mathrm{v}}$ and $v_{\text {eq } i}$ are the volume elasticity and the equilibrium volume of the $i$ th vesicle, respectively. The second term indicates the surface elastic energy of the membrane exerted on individual triangle areas, where $K_{\mathrm{a}}$ and $a_{\text {eq }}$ are membrane-surface elasticity and equilibrium area, respectively. The third term indicates the bending rigidity of the membrane, as exerted on individual vertices, where $K_{\mathrm{c}}$ is a membrane bending rigidity (Julicher 1996; Tsubota 2014). Variable $M_{i}$ denotes the total mean curvature around the $i$ th vertex: $M_{i}=\sum_{j(i)}^{\text {edge }} l_{j} \theta_{j} / 4$, where index $j(i)$ is the $j$ th edge surrounding the $i$ th vertex. Variable $A_{i}$ is the surface area around the $i$ th vertex: $A_{i}=\sum_{j(i)}^{\text {triangle }} a_{j} / 3$, where index $j(i)$ is the $j$ th triangle surrounding the $i$ th vertex.

\subsection{Description of membrane fluidity and turnover}

Cellular membrane has a fluidity, which causes viscous dissipation during membrane deformation. Moreover, membrane molecules are transported between the target vesicle and the reservoir comprising other vesicles within cell. In this model, we regard individual triangular elements as comprising a constant number of membrane molecules. Namely, in the model, the molecules composing the target vesicle is explicitly expressed as the triangular elements, whereas we implicitly express the molecules within the reservoir as a variable. Then, the fluidity can be expressed by a rearrangement of the triangular network (Gompper and Kroll 1998; Noguchi and Gompper 2004, 2005a). Moreover, the turnover can be regarded as a conversion between the triangular elements of the membrane and the number of molecules within the reservoir. Namely, the vesicle size increases when molecules are transferred to and decreases when transferred away from its surface. Therefore, in the model, the fluidity and turnover are expressed from two standpoints: topology and mechanics.

\subsubsection{Spatiotemporal scales of our scope}

To express membrane fluidity and turnover, we argue spatiotemporal scales of our scope.

From a coarse-graining viewpoint, the target vesicle is composed of the large number of triangular elements. Hence, by representing the current number of triangular elements composing the target vesicle by $N_{\mathrm{t}}, N_{\mathrm{t}}$ satisfies the following relationship:

$$
\frac{1}{N_{\mathrm{t}}} \simeq \Delta_{N}
$$

where $\Delta_{N}$ is a positive finite value much smaller than unit. Hence, the spatial scale of vesicle is much larger than that of local triangular elements.

Based on the spatial scale, we consider the relationship of the scales of the numbers of molecules in the target vesicle, reservoir, and the triangular element. Here, the constant number of molecules in the individual triangular elements is represented by $m_{\mathrm{u}}$, the total number of molecules composing the target vesicle by $M_{\mathrm{t}}$, and the total number of molecules in the reservoir by $M_{\mathrm{r}}$. Then, $M_{\mathrm{t}}$ can be denoted by

$M_{\mathrm{t}}=N_{\mathrm{t}} m_{\mathrm{u}}$

Because the membrane molecules are transported between the target vesicle and the reservoir, $M_{\mathrm{t}}$ and $M_{\mathrm{r}}$ are approximately on the same scale:

$M_{\mathrm{t}} \simeq M_{\mathrm{r}}$.

Therefore, $M_{\mathrm{t}}, M_{\mathrm{r}}$, and $m_{\mathrm{u}}$ satisfy the following relationship:

$$
\frac{m_{\mathrm{u}}}{M_{\mathrm{t}}} \simeq \frac{m_{\mathrm{u}}}{M_{\mathrm{r}}} \simeq \Delta_{N}
$$


By focusing on the local spatial scale of individual triangular elements, we consider the timescale of membrane fluidity and turnover, represented by $\tau_{\mathrm{f}}$ and $\tau_{\mathrm{t}}$, respectively. Here, $\tau_{\mathrm{f}}$ is the characteristic time while the local state of individual triangular elements relaxes by the diffusion of membrane molecules, i.e., the viscous dissipation. $\tau_{\mathrm{t}}$ is the characteristic time while the local state of individual triangular elements changes by the transportation of membrane molecules from/to the reservoir. Because the transportation of membrane molecules also requires their local diffusion, the time of local fluidity should be much faster than that of local turnover. Otherwise, the membrane should be locally broken during turnover. Therefore, $\tau_{\mathrm{f}}$ and $\tau_{\mathrm{t}}$ should satisfy the following relationship:

$\frac{\tau_{\mathrm{f}}}{\tau_{\mathrm{t}}} \simeq \Delta_{\tau}$

where $\Delta_{\tau}$ is a positive finit value much smaller than 1 . This local relationship corresponds to the global cellular behaviors: While $\tau_{\mathrm{f}}$ is the period over which the size of the whole cell seems approximately constant, as in blebbing, $\tau_{\mathrm{t}}$ is the period over which the whole cell size dynamically varies, such as during proliferation or differentiation.

Notably, Eq. (11) indicates the timescales of the local dynamics within individual triangular elements, but not the global dynamics of the target vesicle. The timescales of the whole vesicle dynamics caused by the fluidity and turnover, represented by $\tau_{\mathrm{F}}$ and $\tau_{\mathrm{T}}$, can be estimated as

$\tau_{\mathrm{F}}:=M_{\mathrm{t}} /\left(m_{\mathrm{t}} / \tau_{\mathrm{f}}\right)=N_{\mathrm{t}} \tau_{\mathrm{f}}$,

$\tau_{\mathrm{T}}:=M_{\mathrm{t}} /\left(m_{\mathrm{t}} / \tau_{\mathrm{t}}\right)=N_{\mathrm{t}} \tau_{\mathrm{t}}$,

respectively. By comparing the amounts of $\Delta_{N}$ and $\Delta_{\tau}$, from Eqs. (8), (11), (12) and (13), the following relationship is given:

$\left\{\begin{array}{l}\tau_{\mathrm{T}}>\tau_{\mathrm{t}} \geq \tau_{\mathrm{F}}>\tau_{\mathrm{f}} \quad \text { when } \tau_{\mathrm{t}} / \tau_{\mathrm{f}} \geq N_{\mathrm{t}}, \\ \tau_{\mathrm{T}}>\tau_{\mathrm{F}}>\tau_{\mathrm{t}}>\tau_{\mathrm{f}} \quad \text { when } \tau_{\mathrm{t}} / \tau_{\mathrm{f}}<N_{\mathrm{t}}\end{array}\right.$

In case with $\tau_{\mathrm{t}} / \tau_{\mathrm{f}} \geq N_{\mathrm{t}}$, effects of turnover on membrane are immediately relaxed within the whole vesicle. Hence, in the mesoscopic timescale $\tau_{\mathrm{T}}>t>\tau_{\mathrm{f}}$, the vesicle dynamics seem to be dominated by turnover through the number of membrane molecules. On the other hand, in case with $\tau_{\mathrm{t}} / \tau_{\mathrm{f}}<N_{\mathrm{t}}$, effects of local turnover on membrane spend time to relax within the whole vesicle. Hence, in the mesoscopic timescale $\tau_{\mathrm{T}}>t>\tau_{\mathrm{f}}$, the vesicle dynamics seem to be affected by both turnover and fluidity. While the model covers the both cases, this study mainly focuses on the dynamics in case with $\tau_{\mathrm{t}} / \tau_{\mathrm{f}}<N_{\mathrm{t}}$, by assuming large vesicles such as cytomembrane.

\subsubsection{Expression of membrane fluidity}

From a topological viewpoint, the membrane fluidity is modeled by flipping the edges of a couple of neighboring triangles (Gompper and Kroll 1998) (Fig. 2d). Moreover, from Eq. (11), the number of membrane molecules within individual vesicles can be approximately regarded as constant during viscous dissipation. Hence, according to a statistical mechanics, the local state around each edge can be regarded as obeying the canonical ensemble. Thus, the flip frequency of the $i$ th edge, represented by $P_{\mathrm{f} i}$, is given as the following probability:

$P_{\mathrm{f} i}=\frac{1}{\tau_{\mathrm{f}}} \exp \left(-\frac{\Delta_{i} U}{k_{B} T}\right)$

where variable $\Delta_{i} U$ indicates a gap in the total energy before and after flipping the $i$ th edge. Notably, $\tau_{\mathrm{f}}$ reflects the magnitude of membrane viscosity (Noguchi and Gompper 2004, 2005a).

\subsubsection{Expression of membrane turnover}

From a topological viewpoint, the turnover is modeled differently for the increase and decrease in the vesicle size. The increase in the vesicle size is expressed by the local network extension: splitting a couple of neighboring triangles (Fig. $2 b)$. The decrease in the vesicle is expressed by the local network shrinkage: merging a couple of neighboring triangles with their surroundings (Fig. 2c). In these process, the position of a new vertex is determined as the center of the edge shared by the neighboring triangles.

Moreover, from Eq. (11), the local state around each edge, from a statistical-mechanical viewpoint, can be regarded as obeying a grand canonical ensemble. Here, we introduce the difference in effective energy before and after the split and merge around the $i$ th edge, represented by $\Delta_{i} E$, as well as the chemical potential of the reservoir, represented by $\mu_{\mathrm{r}}$. The frequency of the split and merge around the $i$ th edge, represented by $P_{\mathrm{t} i}$, is given by the following probability:

$P_{\mathrm{t} i} \propto \frac{1}{\tau_{\mathrm{t}}} \exp \left(-\frac{\Delta_{i} E \mp 2 m_{\mathrm{u}} \mu_{\mathrm{r}}}{k_{B} T}\right)$,

where the sign $(\mp)$ is negative for splitting and positive for merging. The factor of 2 multiplying the chemical potential originates from the number of triangles transformed by splitting and merging operations. 
a

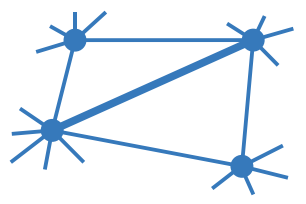

$\checkmark$ Flip

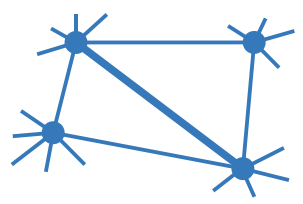

Fluidity

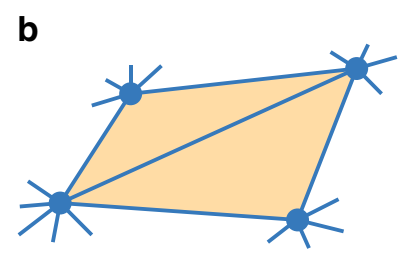

$\checkmark$ Split

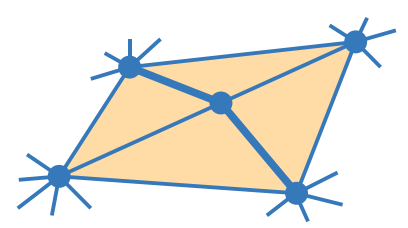

Expansion
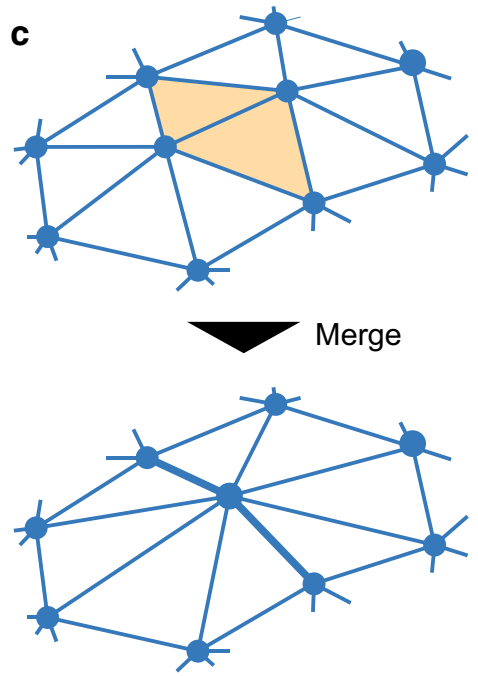

Shrinkage

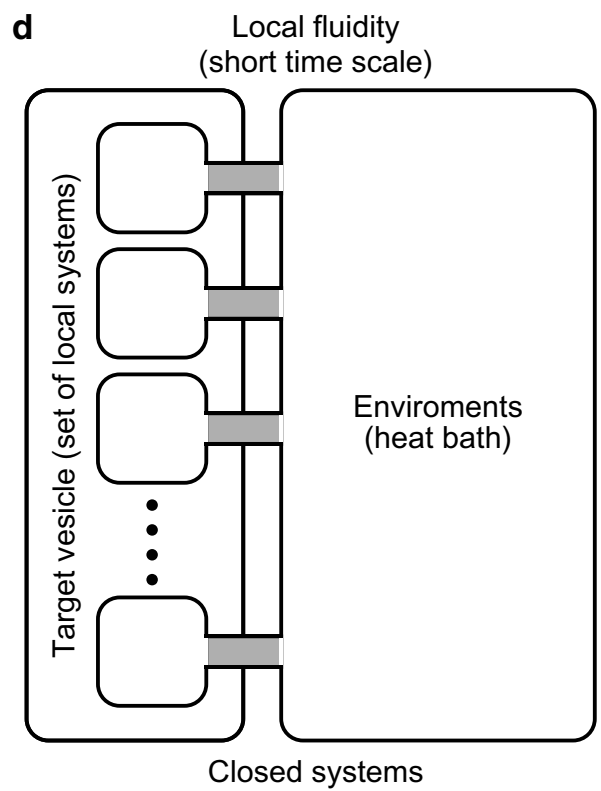

Fig. 2 Topological and thermodynamic model of membrane fluidity and turnover. a Topological flipping operation for expressing membrane fluidity. b Topological splitting operation for expressing membrane expansion. Here, two triangles (yellow triangles in top) are split into four triangles (yellow triangles in bottom) along two edges (thick lines in the bottom). c Topological merging operation for expressing membrane shrinkage. Here two triangles (yellow triangles on top) are merged

\section{Computational simulation of turnover-dependent membrane dynamics}

\subsection{Introducing turnover behavior into the proposed model}

In order to analyze effects of turnover on vesicle dynamics, we simply model the mechanosensing regulation using $\Delta_{i} E$ and $\mu_{\mathrm{r}}$ in Eq. (16).

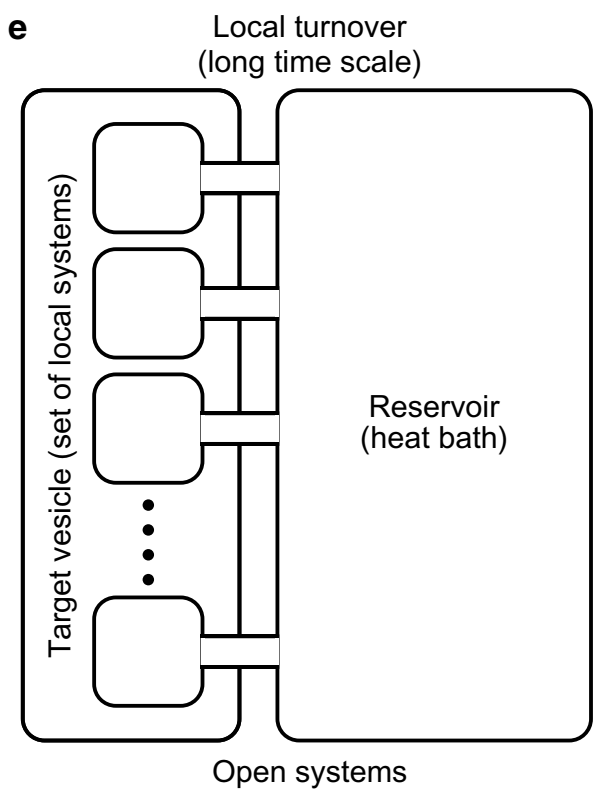

with surrounding triangles by being altered by the addition of two edges (thick lines in bottom). d Thermodynamic system of local membrane fluidity. e Thermodynamic system of local membrane turnover. In d, e, by regarding the target vesicle as a set of local systems, we consider the systems over a short timescale of fluidity and a long timescale of turnover, respectively

Because the local molecular transport depends on the local stress state of membrane, $\Delta_{i} E$ should be a function of vertex positions. As an example, we simply consider the dependency of turnover upon surface-area strain. Because the processes of vesicle fission and fusion require the activities of membrane-associated proteins, $\Delta_{i} E$ should involve an active energy cost in addition to passive energy difference such as the change in membrane curvature energy. Moreover, because the density of molecules composing the membrane 
is likely to be constant, turnover frequency seems approximately proportional to membrane-area strain. Hence, using the first-order approximation, we suppose a linear dependence of turnover upon membrane-area strain. Therefore, by introducing the average surface area of two split or merged triangles adjacent to the $i$ th edge, represented by $\left\langle a_{i}\right\rangle$, we define $\Delta_{i} E$ as follows:

$\Delta_{i} E=\epsilon_{\mathrm{t}}\left\{1 \mp \frac{1}{\gamma_{\mathrm{t}}}\left(\frac{\left\langle a_{i}\right\rangle}{a_{\mathrm{eq}}}-1\right)\right\}$,

where the sign of $(\mp)$ is negative for splitting and positive for merging. Constant $\epsilon_{\mathrm{t}}$ is the energetic cost of molecular turnover. The constant $\gamma_{t}$ is a critical strain for the energetic reduction of molecular turnover.

To simply define $\mu_{\mathrm{r}}$, we consider an isolated system composed of the target vesicle and reservoir. Because membrane transport is actively driven by membrane-associated proteins, the active fluctuation of the turnover is much larger than the thermal fluctuation. Hence, by ignoring osmotic pressure, we focus on the active fluctuation around equilibrium.

For simplification, we assume that the probability density function of $M_{\mathrm{t}}$ as the Gaussian distribution with a mean $M_{\text {eq }}$ and standard deviation $M_{\text {inst }}$ under the condition with $\Delta_{i} E=0$. Here, $M_{\text {inst }}$ can be regarded as the instability of the number of molecules within the target vesicle. This assumption corresponds to defining $\mu_{\mathrm{r}}$ as follows:

$\mu_{\mathrm{r}}=-\frac{\partial G_{\mathrm{r}}}{\partial M_{\mathrm{t}}}, G_{\mathrm{r}}=\frac{k_{B} T}{12 m_{\mathrm{u}} M_{\mathrm{inst}}^{2}}\left|M_{\mathrm{t}}-M_{\mathrm{eq}}\right|^{3}$,

where $G_{\mathrm{r}}$ is the Gibbs free energy of the reservoir. Here, we introduce the current number of molecules within the vesicle, represented by $M_{\mathrm{t}}$, as a continuum quantity. Then, to convert the triangular elements into molecules, we substitute Eq. (8).

By assuming the mass conservation law in the total number of molecules within cell, represented by $M_{\mathrm{tt}}:=M_{\mathrm{r}}+M_{\mathrm{t}}$, $M_{\mathrm{tt}}$ is constant. Therefore, $\mu_{\mathrm{r}}$ can be rewritten as follows:

$\mu_{\mathrm{r}}=\frac{\partial G_{\mathrm{r}}}{\partial M_{\mathrm{r}}}, G_{\mathrm{r}}=\frac{k_{B} T}{12 m_{\mathrm{u}} M_{\mathrm{inst}}^{2}}\left|M_{\mathrm{r}}-M_{\mathrm{req}}\right|^{3}$,

where $M_{\text {req }}$ is the equilibrium number of molecules in the reservoir: $M_{\mathrm{req}}=M_{\mathrm{tt}}-M_{\mathrm{eq}}$. Namely, the employed assumption of $M_{\mathrm{t}}$ corresponds to the second-order approximation of the active fluctuation of the number of molecules within the reservoir under equilibrium.

\subsection{Non-dimensionalization and parameter setting}

To solve Eq. (1), parameter values are normalized to have unit length $(l)$, unit time $(\tau)$, unit number of molecules $(m)$, and unit energy $\left(k_{B} T\right)$. Here, $l, \tau$, and $m$ are set as $l=\left(a_{\mathrm{eq}}\right)^{\frac{1}{2}}, \tau=0.1 \eta a_{\mathrm{eq}} / k_{B} T$, and $m=m_{\mathrm{u}}$. Hereafter, physical parameters are described as dimensionless values. In case where a specific membrane is focused upon, the physical parameters employed in the simulations can be determined based on those measured by experiments. By assuming the system temperature to be $310 \mathrm{~K}$, unit energy $k_{B} T$ becomes $4.3 \times 10^{-20} \mathrm{~J}$. Based on this, the values of $K_{\mathrm{c}}$ employed in this study correspond to $4.3 \times 10^{-20}$ $4.3 \times 10^{-19} \mathrm{~J}$. These values have the similar range of those of the dimyristoylphosphatidylcholine (DMPC), the plant thylakoid lipid digalactosyldiacylglycerol (DGDG), and other general lipid membranes (Duwe et al. 1990; Engelhardt et al. 1985; Schneider et al. 1984; Mutz and Helfrich 1990; Evans and Rawicz 1990; Kummrow and Helfrich 1991).

To establish that the proposed model successfully recapitulates turnover-dependent membrane dynamics, several parameters are varied, such as $K_{\mathrm{c}}$ and $\tau_{\mathrm{t}}$. The state under the initial condition is set as a single vesicle composed of 1000 triangles, which are equilibrated under the condition $K_{\mathrm{c}}=10$. The equilibrium volume of the vesicle is set to $v_{\text {eq }}=2527$, which corresponds to the volume of the vesicle with the area $1000 a_{\mathrm{eq}}$ and sphericity 0.85 . The setting of the geometric constraint $l_{\text {rep }}$ in Eq. (5) is described in Appendix A. Moreover, to set physical parameters, the force balance among individual energy terms in Eqs. (5) and (6) is taken into account, as described in Appendix B. Numerical implementation and calculation is described in Appendix C. All model parameters are shown in Table 1.

\subsection{Proposed model successfully recapitulates turnover-dependent membrane dynamics}

To establish whether the proposed model successfully recapitulates turnover-dependent membrane dynamics, we simulate vesicle dynamics in case with and without membrane turnover $\left(\tau_{t}=1.0\right.$ and $\left.+\infty\right)$ (Fig. 3). In the case without membrane turnover, the vesicle is slightly deformed by fluctuations while maintaining its surface area (Fig. 3a, c). On the other hand, in the case with turnover, the vesicle is significantly deformed as its surface expands (Fig. 3b, c). Hence, the large deformation is permitted by the surface-area extension (Fig. 3c). Moreover, for a long timescale, the total surface area and number of molecules reached the plateau (Fig. 3c, d). This tendency seems independent on the values of the bending rigidity $K_{\mathrm{c}}$ and transport instability $M_{\text {inst }}$; meanwhile, we could not observe the plateau in the time range of our simulations in case with large $M_{\text {inst }}$. Importantly, the extension is caused by the increase in the number of molecules within the vesicle (Fig. 3d), but not by elastic deformation. In the process of this expansion, the number of molecules within the vesicle gradually increases in a stochastic manner (Fig. 3e). These results suggested that the proposed model 
Table 1 Model parameters

\begin{tabular}{|c|c|c|}
\hline Symbol & Value & Description \\
\hline \multicolumn{3}{|c|}{ Parameters for physical behaviors } \\
\hline$K_{\mathrm{V}}$ & $1.0 \times 10^{4}$ & Volume elasticity of vesicle \\
\hline$K_{\mathrm{a}}$ & 50 & Surface elasticity of membrane \\
\hline$K_{\mathrm{c}}$ & $1.0-10$ & Bending rigidity of membrane \\
\hline$\kappa_{\text {act }}$ & $1.0-3.0$ & $\begin{array}{l}\text { Active surface energy on } \\
\text { membrane }\end{array}$ \\
\hline$v_{\mathrm{eq}}$ & 2527 & Equilibrium volume of vesicle \\
\hline$\tau_{\mathrm{f}}$ & $1.0 \times 10^{-2}$ & $\begin{array}{l}\text { Characteristic time of } \\
\text { membrane fluidity }\end{array}$ \\
\hline$\tau_{\mathrm{t}}$ & $1.0,+\infty$ & $\begin{array}{l}\text { Characteristic time of } \\
\text { membrane turnover }\end{array}$ \\
\hline$M_{\mathrm{eq}}$ & $1.0 \times 10^{3}$ & $\begin{array}{l}\text { Equilibrium number of } \\
\text { molecules within vesicle }\end{array}$ \\
\hline$M_{\text {inst }}$ & $10-1.0 \times 10^{3}$ & $\begin{array}{l}\text { Instability of the number of } \\
\text { molecules within vesicle }\end{array}$ \\
\hline$\epsilon_{\mathrm{t}}$ & $1.0 \times 10^{-1}$ & $\begin{array}{l}\text { Energetic cost of molecular } \\
\text { turnover }\end{array}$ \\
\hline$\gamma_{\mathrm{t}}$ & $5.0 \times 10^{-2}$ & $\begin{array}{l}\text { Critical strain for energetic } \\
\text { reduction of molecular } \\
\text { turnover }\end{array}$ \\
\hline \multicolumn{3}{|c|}{ Parameters for computational calculation } \\
\hline$K_{\mathrm{r}}$ & $1.0 \times 10^{3}$ & Repulsive modulus \\
\hline$\Delta t_{\mathrm{M}}$ & $1.0 \times 10^{-3}$ & $\begin{array}{l}\text { Time step of numerical } \\
\text { integration }\end{array}$ \\
\hline
\end{tabular}

successfully recapitulates the turnover-dependent membrane dynamics.

\subsection{Molecular turnover adaptively facilitates vesicle deformation}

Next, to investigate the effects of molecular turnover upon membrane deformation, we analyze the effects of the bending rigidity $K_{\mathrm{c}}$ and the instability modulus of the number of molecules within vesicle $M_{\text {inst }}$ (Fig. 4). To focus on the fundamental effects of turnover on membrane dynamics, we set $U_{\text {act }}=0$ in Eq. (4) by assuming a simple membrane behavior.

The vesicle dynamics are found to drastically vary with respect to $K_{\mathrm{c}}$ and $M_{\text {inst }}$ (Fig. 4a, b). In the case with small $M_{\text {inst }}$, the vesicle slightly deforms while maintaining its surface area. On the other hand, in case with large $M_{\text {inst }}$, the vesicle morphology drastically varies as it extends or shrinks with respect to $K_{\mathrm{c}}$. In case with small $K_{\mathrm{c}}$, the vesicle deforms to be lobate as its surface area increases. In case with large $K_{\mathrm{c}}$, the vesicle deforms to be spherical as its surface area decreases.

To analyze effects of $K_{\mathrm{c}}$ on membrane shape, we measured the averaged local Gaussian curvature over every vertex, which is estimated from a set of the surrounding vertices. Notably, the averaged local Gaussian curvature cannot be conserved in the defiance of the Gauss-Bonnet theorem but dynamically vary. This is because the curvature is locally defined at individual vertices, whose number dynamically varies by turnover. Interestingly, the dependence of the averaged local Gaussian curvature on $M_{\text {inst }}$ changes directions at three areas; positive in cases $K_{\mathrm{c}} \lesssim 2$, negative in cases $2 \lesssim K_{\mathrm{c}} \lesssim 20$ and positive $20 \lesssim K_{\mathrm{c}}$ (Fig. 4b). On the other hand, the number of membrane molecules is inversely proportional to $K_{\mathrm{c}}$ independent on $M_{\text {inst }}$. These behaviors can be simply explained by the geometric constraint imposed by the volume-area balance; the surface must be finely folded in cases with large area $\left(K_{\mathrm{c}} \lesssim 2\right)$, laminarly flatted in cases with middle area $\left(2 \lesssim K_{\mathrm{c}} \lesssim 20\right)$ and smoothly spherical in cases with small area ( $20 \lesssim K_{\mathrm{c}}$ ). Therefore, the resulting membrane morphologies are regulated by the number of membrane molecules through turnover.

The turnover is dependent on $K_{\mathrm{c}}$ because it reduces the local residual stress generated by the global force balance: in the case with low $K_{\mathrm{c}}$, the membrane-surface area tends to expand because of the thermal fluctuation force. As $K_{\mathrm{c}}$ increases, the membrane-surface area tends to decrease to minimize its bending energy. Therefore, the strain of the membrane-surface area $\left\langle a_{i}\right\rangle / a_{\mathrm{eq}}$ is inversely proportional to the bending rigidity, $K_{\mathrm{c}}$. By sensing local stress as Eq. (17), the molecular turnover is biased to cause expansion or shrinkage. These results suggest that the turnover serves to adaptively facilitate membrane deformation depending upon the membrane-stress state.

\subsection{Molecular turnover permits autonomous cell migration}

Finally, to demonstrate the use of the model, we simulated cellular dynamics driven by an intracellular active force of lining cytoskeleton on membrane. To exert this force, we introduce a locally biased cortical surface energy as follows.

$U_{\text {act }}=\sum_{i}^{\text {triangle }} \frac{\kappa_{\text {act }}\left(1+\cos \phi_{i}\right)}{2} a_{i}$

where $\phi_{i}$ is the constant defined at each time step as the angle between the $x$-axis and the vector from the center of the vesicle to the center of the $i$ th triangle. This function is similar to that used in expressing the active energy on cells during collective migration (Sato et al. 2015).

As a result, in case without turnover, the velocity of migration drastically decreases (Fig. 5a, c). On the other hand, in case with turnover, the cell dynamically migrates along the $x$-axis (Fig. $5 \mathrm{~b}, \mathrm{c}$ ). This is because the active energy in Eq. (20) drives the transport of molecules from the rear 

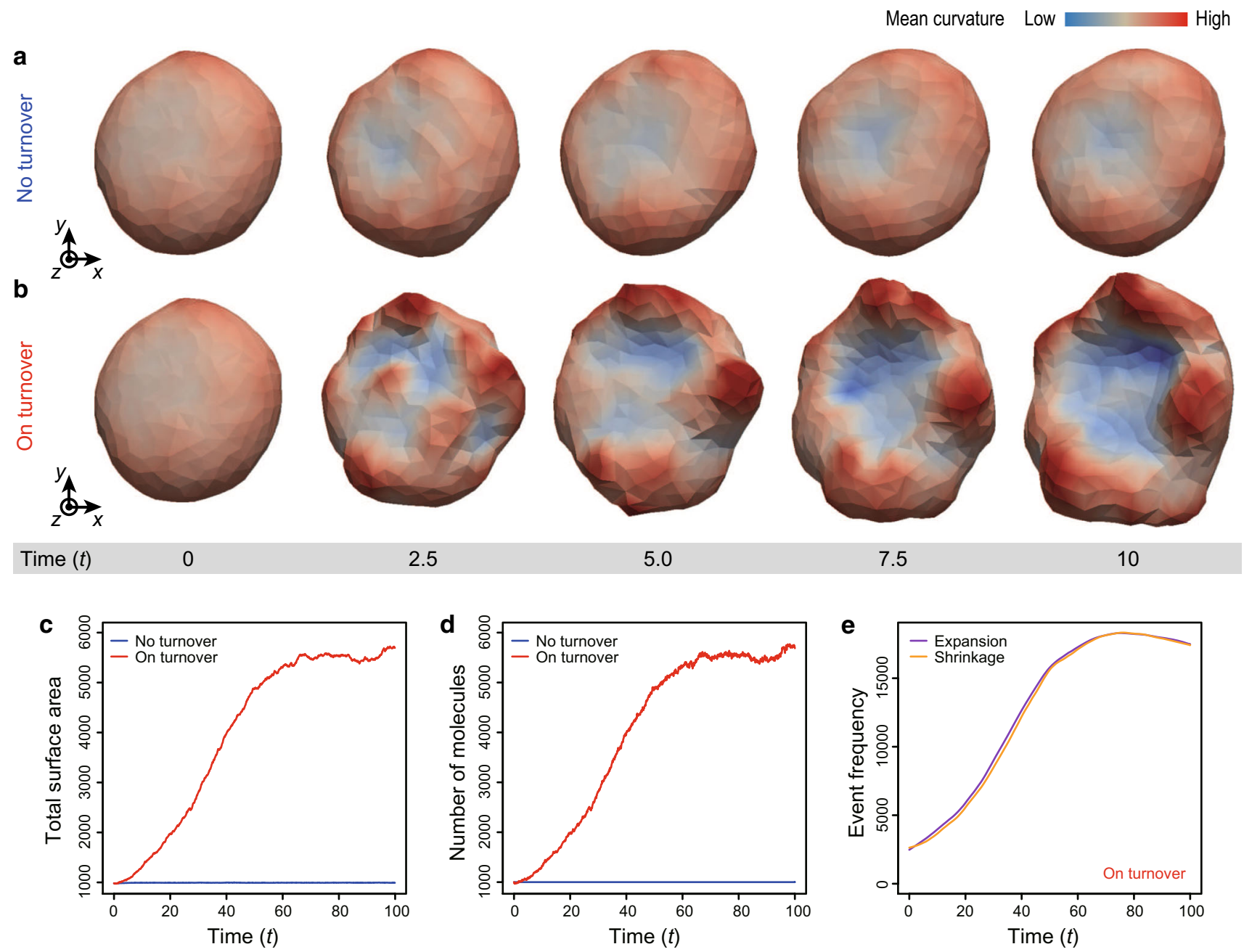

2.5

5.0

7.5

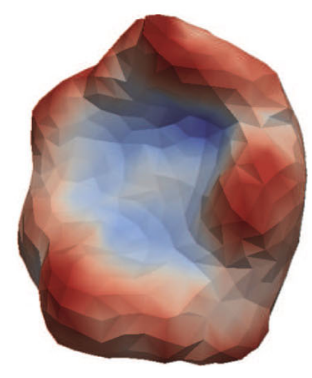

10
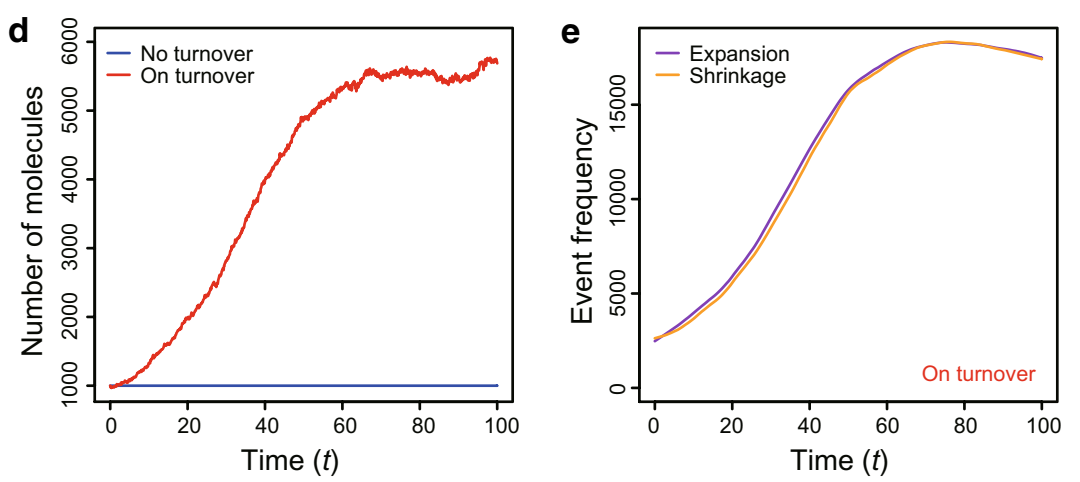

Fig. 3 Turnover-dependent dynamics of cellular membranes. a Deformation process of the vesicle in the case without turnover $\left(\tau_{\mathrm{t}}=+\infty\right)$. $\mathbf{b}$ Deformation process of the vesicle in the case with turnover $\left(\tau_{\mathrm{t}}=1.0\right)$. Vesicles are colored according to their local mean curvature. The dynamic process of $\mathbf{b}$ is also shown in Supplementary Movie 1. $\mathbf{c}$ Total surface area of the vesicle as a function of time $t$. d The number of membrane molecules as a function of time $t$. e Frequencies of expansion and shrinkage as functions of time $t$. The dynamics of $\mathbf{a}$ are calculated under condition $K_{\mathrm{c}}=3.0$. The dynamics of $\mathbf{b}$ are calculated under conditions $K_{\mathrm{c}}=3.0$ and $M_{\text {inst }}=1000$ to the front through the turnover (Fig. 5d). This result suggests that cells can migrate even without any traction force on membrane, with turnover playing a crucial role in driving migration. Notably, while the migration can be observed in the wide range of $K_{\mathrm{c}}$ and $M_{\text {inst }}, K_{\mathrm{c}}$ must be high to maintain the spherical cell shape as described in Appendix E.

Notably, because the force from the energy in Eq. (20) is internal within cell but not external, the force is balanced within cell at each time. On the other hand, because $\phi_{i}$ dynamically varies with time under membrane deformation, $U$ becomes non-conservative so as to set the system into non-equilibrium. Such non-conservative energy function has been known as to generate active cell movements (Sato et al. 2015). Moreover, the stress-dependency of the turnover in
Eq. (16) breaks the detail balance of molecular transport. Thus, despite the force balance within cell, this model can generate cell migration in a physically consistent manner.

In biological systems, there are a lot of types of cell migration such as single and collective cell migration in wound healing, morphogenesis and cancer invasion (Friedl and Wolf 2003). Importantly, while mechanism of the resulting process is a kind of Brownian ratchet, it differs from the mechanism of the well-known single-cell migration. In this mechanism, the front extension of the cell initiates migration: It extends ahead by cytoskeletal polymerization and the rear follows it by cytoskeletal contraction. On the contrary, in our model, localized active tension at the rear initiates migration by directionally transporting molecules to the front. Notably, our model does not conflict with the mechanism of the single- 
a

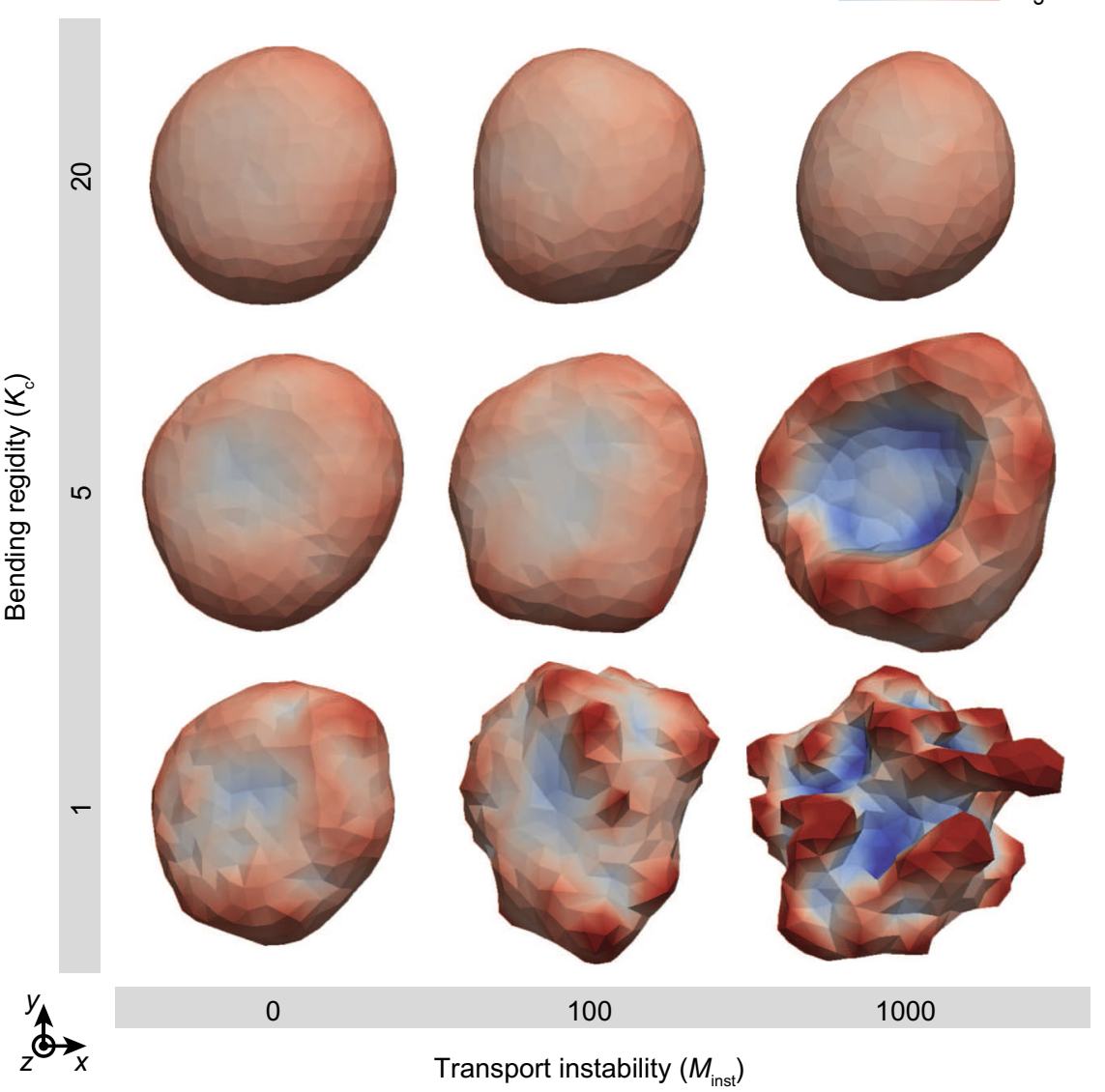

b
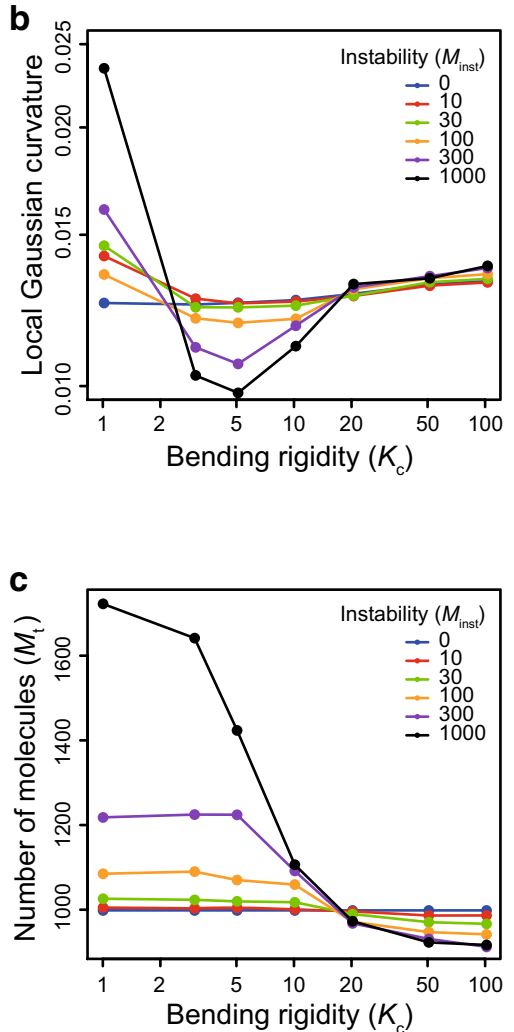

Fig. 4 Effect of membrane turnover on membrane deformation. a Phase diagram of vesicle morphology for bending rigidity $K_{\mathrm{c}}$ and transport instability $M_{\text {inst }}$ at $t=10$. Vesicles are colored based on the local mean curvature. b Averaged local Gaussian curvature as a function of bending rigidity $K_{\mathrm{c}}$ with respect to transport instability $M_{\mathrm{inst}}$ at $t=10$. c The number of molecules as a function of bending rigidity $K_{\mathrm{c}}$, with respect to transport instability $M_{\text {inst }}$ at $t=10$. These dynamics are calculated under the condition of $\tau_{\mathrm{t}}=1.0$ cell migration. Therefore, turnover may contribute to cellular migration as a main or secondary process.

\section{Discussion}

In this study, by making several physical assumptions, we integrally formulated the fluidity and turnover of a cellular membrane in a physically consistent manner. In the computational simulations, the cellular vesicles actively deform by the turnover of the membrane molecules (Fig. 3). During deformations, the triangular property was maintained to satisfy the modeling assumptions as in Appendix D. Moreover, based on the dependence of turnover upon the stress state, vesicle morphology drastically differed with the bending rigidity (Fig. 4); a smooth sphere was obtained under high rigidity, and a lobate vesicle was obtained under low rigidity. Furthermore, based on directional molecular transport by turnover, the localized active tension on the membrane drove the cellular migration of the vesicle (Fig. 5). From these results, the proposed model successfully recapitulated the turnover-dependent dynamics of three-dimensional cellular membranes.

In the computational simulations, the turnover behavior described in Eq. (16) was simply provided under the following assumptions: (i) that the energetic cost for molecular transport, $\Delta_{i} E$, depended on the local strain of the membrane area, as shown in Eq. (17) and (ii) that the chemical potential $\mu_{\mathrm{t}}$ stabilized the number of molecules in the reservoir, as shown in Eq. (18). In general, these assumptions were not necessary for the proposed model. We emphasize that the functions of $\Delta_{i} E$ and $\mu_{\mathrm{r}}$ can be entirely arbitrary.

Although the cellular mechanical property was expressed simply in these simulations, as described in Eq. (6), it can be expressed in more detail. For example, in cells with rich lining cytoskeletons, cell membranes could have non-negligible longitudinal and transverse elasticity, which may be important for their deformations (Heinrich et al. 2001). Moreover, 

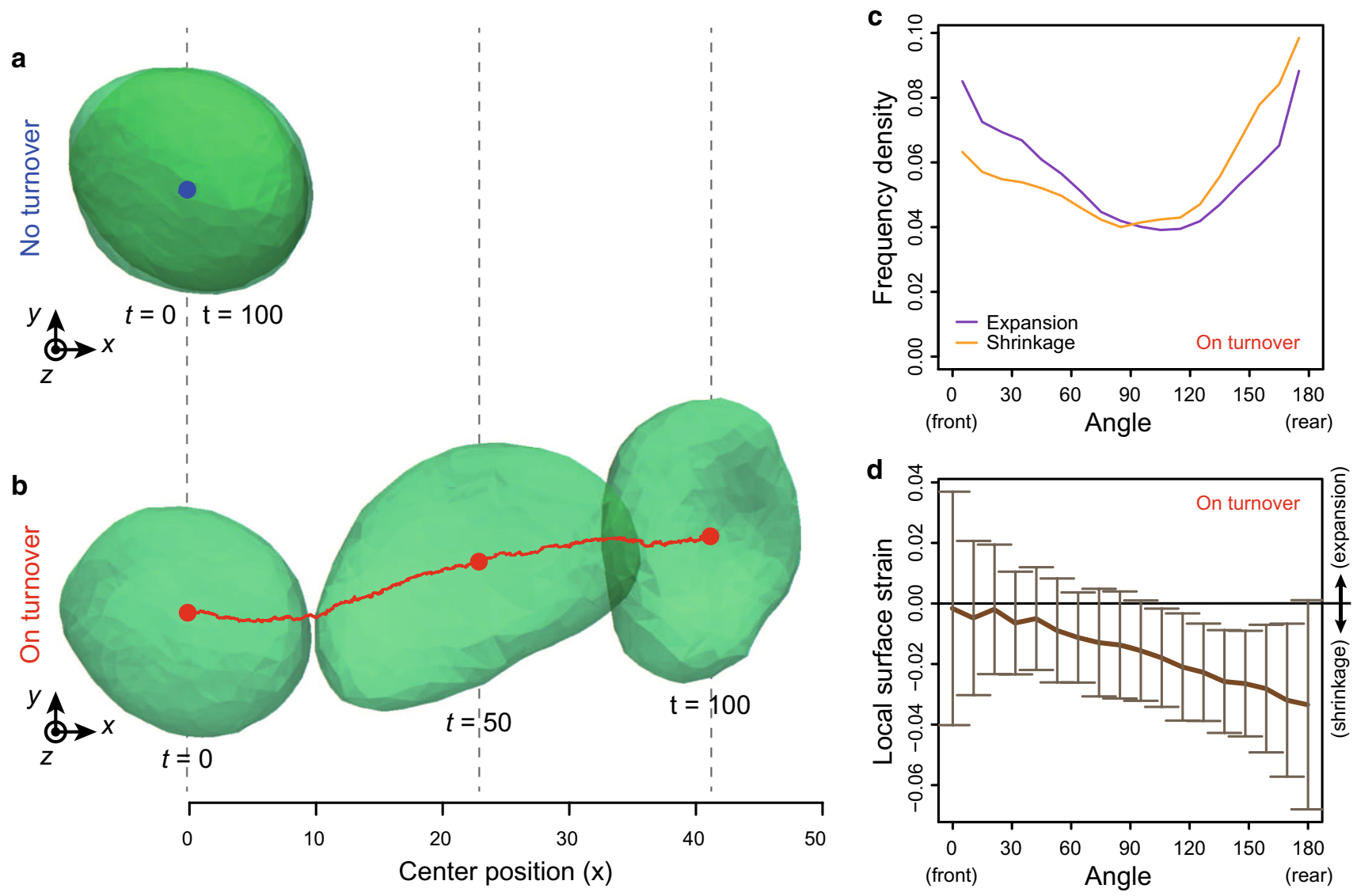

Fig. 5 Effect of localized active tension on the membrane dynamics throughout turnover. a Vesicle dynamics without turnover $\left(\tau_{\mathrm{t}}=+\infty\right)$. b Vesicle dynamics with turnover $\left(\tau_{\mathrm{t}}=1.0\right)$. The dynamic process of b is also shown in Supplementary Movie 2. c Normalized frequency densities of expansion and shrinkage in case with turnover as a function of the angle to the event site. $\mathbf{d}$ Local strain of the membrane surface in case with turnover as a function of the angle. The angle in $\mathbf{c}, \mathbf{d}$ is

during blebbing, the actin cytoskeleton lining membrane is locally broken, which causes a spatiotemporal inhomogeneity in membrane rigidity (Manakova et al. 2016). These details can be reflected by choosing a proper effective energy function, $U_{\text {eff }}$ in Eq. (6).

In principle, detailed expressions for turnover at a molecular scale are limited in the proposed model; for example, the adhesion process of membrane-associated proteins cannot be directly expressed. Such behaviors at the molecular scale must be instead coarse-grained into those at the minimum scale of the proposed model. For example, the dependence of protein adhesion upon lipid behavior can be expressed by $\Delta_{i} E$ as a dependence of the turnover upon membrane strain. Moreover, the biased adhesion of proteins to lipids can be expressed by $\mu_{\mathrm{r}}$ as directional molecular transport. Thus, by designing $\Delta_{i} E$ and $\mu_{\mathrm{r}}$, the proposed model can be applied to various behaviors of turnover. defined as that between the $x$-axis and the vector from the center of the vesicle to the event site. In $\mathbf{c}$, the frequency density is calculated as the local frequency per the surface area of sphere within the scope angle. In d, the local surface strain is calculated as the strain of individual triangular elements, and the solid line and bar indicate the average and standard deviation within the scope angle, respectively. These dynamics are calculated under the condition $K_{\mathrm{c}}=10, M_{\text {inst }}=10$, and $K_{\text {act }}=3.0$

The proposed model will help researchers understand the mechanics of 3D cellular dynamics involving membrane turnover, particularly the causal relationships between single-cell dynamics and the underlying molecular transport. The proposed model is a powerful approach for addressing the manner in which molecular turnover affects 3D cellular dynamics at a subcellular scale. Understanding these functions of turnover is necessary for better understanding cellular dynamics, and this will be useful as fundamental knowledge for controlling these dynamics in bioengineering. Moreover, by combining the proposed model at a subcellular scale with the models at a multicellular scale (Okuda et al. 2013, 2015), it will be possible to predict comprehensive cellular dynamics from molecules to tissues. Therefore, the proposed model will contribute to exploring the exploration of the frontiers of cellular biomechanics. 


\section{Conclusion}

To analyze the effect of molecular turnover on threedimensional deformations of cellular membranes, we proposed a new computational model for simulating the turnoverdependent dynamics of three-dimensional cellular membranes. The proposed model successfully simulate turnoverdependent membrane dynamics, and suggested the roles of turnover to drive the adaptive deformation and directional migration of vesicles. These results illustrate the importance of turnover in the dynamic deformations of cellular membranes in addition to the use of the proposed model for exploring general effects of molecular turnover on cellular dynamics.

Acknowledgements We appreciate Dr. Masashi Tachikawa (RIKEN, Japan) and Prof. Ken-ichi Tsubota (Chiba University, Japan) for their valuable comments.

Funding This work was partially supported by JSPS KAKENHI Grant No. 15K14534, Japan. This work was partially supported by JST/PRESTO Grant No. JPMJPR16F3, Japan.

\section{Compliance with ethical standards}

Conflict of interest There is no financial and personal relationship with other people or organizations that could inappropriately influence this work.

Open Access This article is distributed under the terms of the Creative Commons Attribution 4.0 International License (http://creativecomm ons.org/licenses/by/4.0/), which permits unrestricted use, distribution, and reproduction in any medium, provided you give appropriate credit to the original author(s) and the source, provide a link to the Creative Commons license, and indicate if changes were made.

\section{A Appendix}

\section{A Setting geometric constraint}

Because the constraint energy $U_{\mathrm{cc}}$ limits the distances between vertices, it can reduce the topological flexibility of the triangular meshwork. To determine the value of $l_{\text {rep }}$, we consider a $n$-side polygon surrounding a central vertex, wherein individual triangles have the same area $a_{\mathrm{eq}}$. Then, the minimum lengths of edges of individual triangles can be given as a function of $n$. Then, to permit topological flexibility between 4 - and 8 -sided polygons (Fig. 6), we set $l_{\text {rep }}$ to be the minimum length of all of their edges as follows:

$l_{\text {rep }}=2\left(\frac{a_{\mathrm{eq}}}{\tan (7 \pi / 16)}\right)^{\frac{1}{2}}$.

Notably, because triangles can deform out-of-plane, the constraint condition does not rigidly limit the number of sides.

\section{B Energetic force balance}

From a physical viewpoint, the magnitudes of several parameters in Eq. (4) are related. First, because cellular membranes cannot cross over each other, the repulsive force in Eq. (5) should be enough large to act as a constraint. Second, because the cytoplasm and lipid membrane generally behave as incompressible liquids, the volume elasticity of the vesicles and the area elasticity of the membranes should be much larger than the other physical parameters. Moreover, because cellular membranes are constructed mainly from lipids, the bending rigidity of the membrane in Eq. (6) should be much smaller than the area elasticity of membrane. Therefore, individual forces exerted on vertices, represented by $-\nabla U$, should satisfy the following force balance:

$\left|\nabla U_{\mathrm{cc}}\right| \gg\left|\nabla U_{\mathrm{v}}\right| \simeq\left|\nabla U_{\mathrm{a}}\right| \gg\left|\nabla U_{\mathrm{c}}\right|$,

where $U_{\mathrm{v}}, U_{\mathrm{a}}$, and $U_{\mathrm{c}}$ are the first, second, and third terms of $U_{\text {eff }}$, respectively. Several constant parameters in Eq. (4) are set to satisfy Eq. (22).

\section{Numerical implementation and calculation}

Monte Carlo simulations for sampling in Eqs. (15) and (16) were numerically conducted using the heat-bath algorithm at each interval of $\Delta t_{\mathrm{M}}$. Time integration of Eq. (1) was numerically performed using the Euler method with a variable time step of $\Delta t_{\mathrm{E}}\left(\leq \Delta t_{\mathrm{M}}\right)$, which is dynamically determined to suppress the maximum vertex displacement. Because the frequencies of flip, split, and merge are enough low, the system state statistically reaches equilibrium during each interval of $\Delta t_{\mathrm{M}}$. Because the error in the numerical integration $(O(\Delta t))$ is much smaller than the magnitude of thermal noise $\left(O\left(\Delta t^{\frac{1}{2}}\right)\right)$, we employed the first-order Euler method.
Fig. 6 Regular in-plane polygons with triangles of the same area, $a_{\mathrm{eq}}$, for determining the repulsive distance, $l_{\text {rep }}$

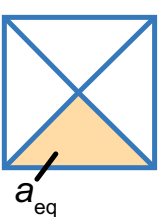

4-side

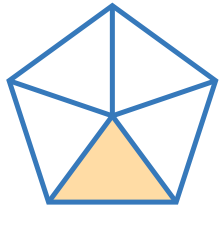

5-side

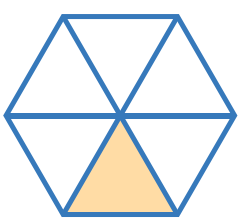

6-side

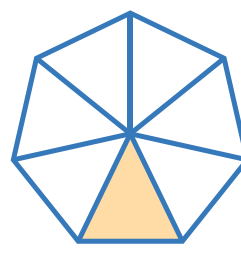

7-side

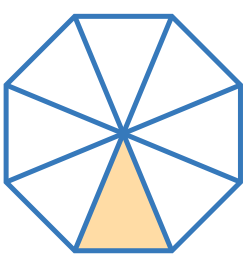

8-side 

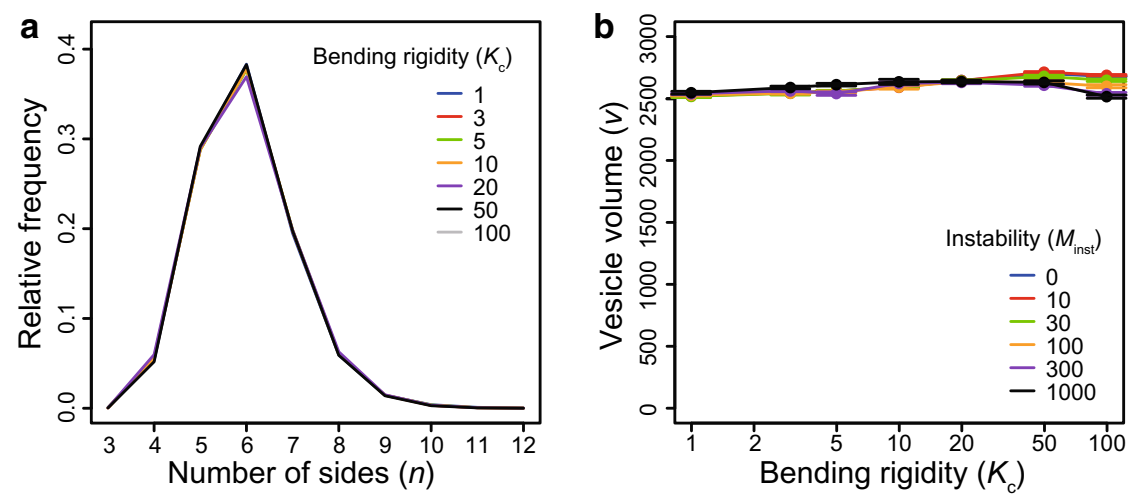

Fig. 7 Membrane and triangular property. a Probability distribution of the number of triangular sides, $n$. $\mathbf{b}$ Vesicle volume, $v$, as a function of bending rigidity, $K_{\mathrm{c}}$. c Probability distribution of triangular area, $a$. These probability distributions are calculated from the dynamics within the range of $9 \leq t \leq 10$. The dynamics in $\mathbf{a}, \mathbf{c}$ are calculated under the conditions $\tau_{\mathrm{t}}=1.0$ and $M_{\mathrm{inst}}=1000$. The dynamics in $\mathbf{b}$ are calculated under the condition $\tau_{\mathrm{t}}=1.0$

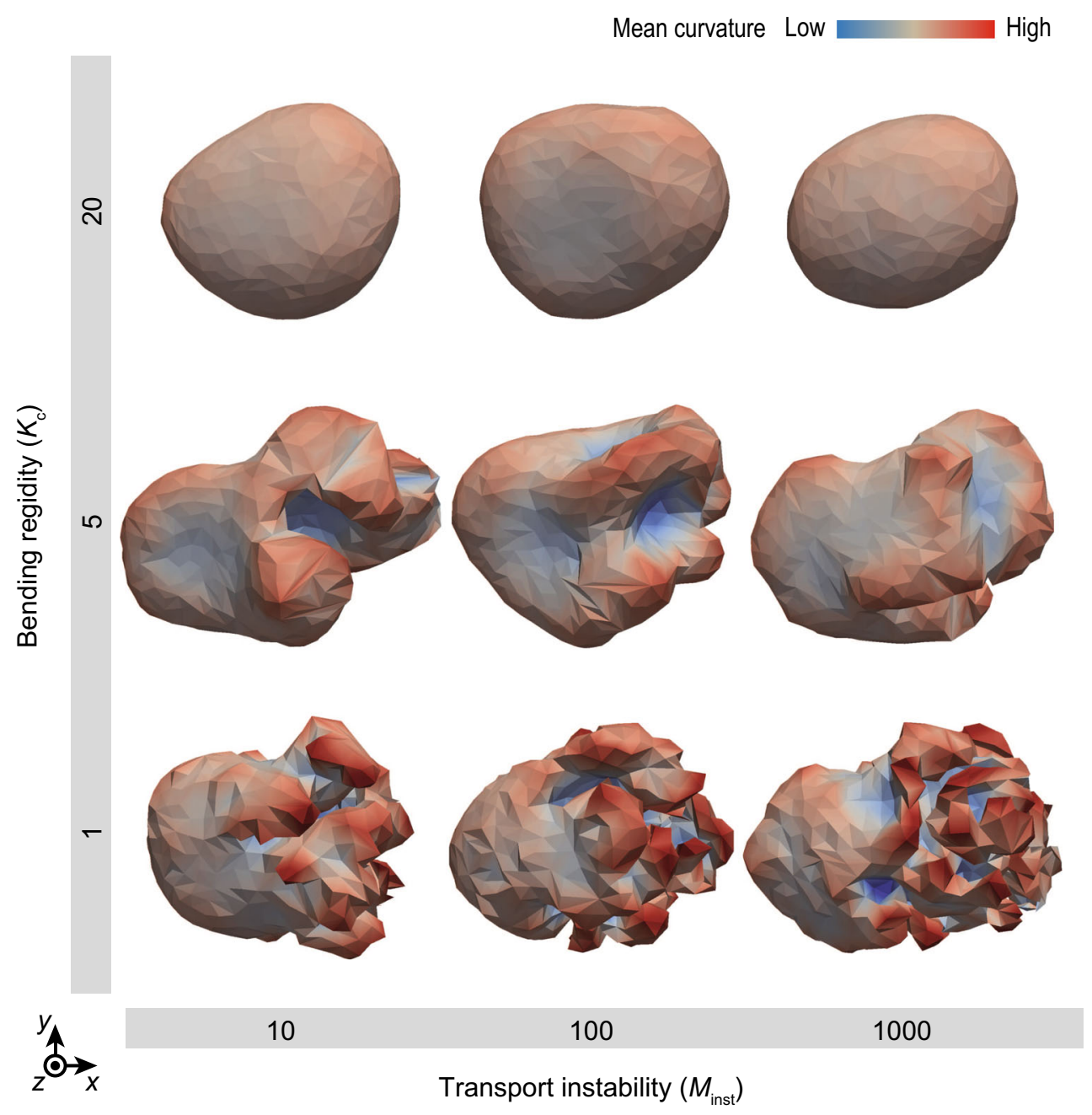

Fig. 8 Effect of membrane turnover on cell morphology during migration. Cell morphologies at $t=5$ are shown with respect to the bending rigidity $K_{\mathrm{c}}$ and transport instability $M_{\text {inst }}$. Vesicles are colored based on the local mean curvature. These dynamics are calculated under the condition of $\tau_{\mathrm{t}}=1.0$ and $K_{\text {act }}=1.0$ 
All experiments were performed on a cluster computer comprising 12 nodes with $2.9 \mathrm{GHz}$ Intel Xeon dual processors and 64 GB RAM.

\section{Validation of assumptions}

In the proposed model, we made several assumptions: (i) that the timescales of membrane fluidity and turnover are related as in Eq. (11); (ii) that there is topological flexibility for membrane fluidity, and (iii) that the force balance is given by Eq. (22). Preassumption (i) is satisfied because it is given by the set of parameters. On the other hand, preassumptions (ii) and (iii) are non-trivial because they result from the dynamic process of membrane deformation. To ascertain whether these preassumptions are satisfied in the results, we analyze properties of the membrane and triangles. Firstly, to test the membrane fluidity, we analyze the probability distribution of the number of sides, which is broad from 4 to 9 (Fig. 7a). Hence, in the case where $l_{\text {rep }}$ is set as Eq. (21), the constraint energy permits the topological flexibility of the meshwork. Secondly, to test the balance of the individual energetic forces, we analyze the probability distributions of vesicle volume and triangular area. These distributions approximately equal those determined by the first and second terms of Eq. (6) (Fig. 7b, c), respectively. Hence, the preassumed force balance of Eq. (22) is satisfied. Moreover, from the distribution of triangular area in Fig. 7c, the discrete size of the triangular meshwork is approximately maintained. These results warrant certain predictions of the model from a physical viewpoint.

\section{E Effect of membrane turnover on cell morphology during migration}

To analyze the effect of molecular turnover on migration, we simulated cellular dynamics driven by the intracellular active force, described by Eq. (20). Figure 8 shows the cell morphologies during migration with respect to $K_{\mathrm{c}}$ and $M_{\text {inst }}$. The results suggested that $K_{\mathrm{c}}$ must be high to maintain the cell shape to be spherical. Otherwise, the cell surface strongly undulated.

\section{References}

Atilgan E, Sun SX (2007) Shape transitions in lipid membranes and protein mediated vesicle fusion and fission. J Chem Phys 126(9):03B604

Boal D, Rao M (1992) Topology changes in fluid membranes. Phys Rev A 46(6):3037-3045

Chiu S, Clark M, Balaji V, Subramaniam S, Scott H, Jakobsson E (1995) Incorporation of surface tension into molecular dynamics simula- tion of an interface: a fluid phase lipid bilayer membrane. Biophys J 69(4):1230-1245

Duwe H, Kaes J, Sackmann E (1990) Bending elastic moduli of lipid bilayers: modulation by solutes. J Phys 51(10):945-961

Engelhardt H, Duwe H, Sackmann E (1985) Bilayer bending elasticity measured by fourier analysis of thermally excited surface undulations of flaccid vesicles. J Phys Lett 46(8):395-400

Evans E, Rawicz W (1990) Entropy-driven tension and bending elasticity in condensed-fluid membranes. Phys Rev Lett 64(17):2094

Friedl P, Wolf K (2003) Tumour-cell invasion and migration: diversity and escape mechanisms. Nat Rev Cancer 3(5):362-374

Ghosh P, Singh U (1992) Liposome shrinkage and swelling under osmotic-diffusional stress: evaluation of kinetic parameters from spectrophotometric measurements. Biochem Biophys Acta 1110(1):88-96

Gompper G, Kroll D (1997) Network models of fluid, hexatic and polymerized membranes. J Phys Condens Matter 9(42):8795-8834

Gompper G, Kroll D (1998) Membranes with fluctuating topology: Monte carlo simulations. Phys Rev Lett 81:2284

Gompper G, Kroll D (2004) Statistical mechanics of membranes and surfaces, 2nd edn. World Scientific, Singapore

Grant B, Donaldson J (2009) Pathways and mechanisms of endocytic recycling. Nat Rev Mol Cell Biol 10(9):597-608

Heinrich V, Ritchie K, Mohandas N, Evans E (2001) Elastic thickness compressibilty of the red cell membrane. Biophys J 81(3):14521463

Heller H, Schaefer M, Schulten K (1993) Molecular dynamics simulation of a bilayer of 200 lipids in the gel and in the liquid crystal phase. J Phys Chem 97(31):8343-8360

Ho J, Baumgärtner A (1990) Simulations of fluid self-avoiding membranes. Europhys Lett 12:295-300

Julicher F (1996) The morphology of vesicles of higher topological genus: conformal degeneracy and conformal modes. J Phys II 6:1797-1824

Keren K (2011) Cell motility - the integrating role of the plasma membrane. Eur Biophys J 40(9):1013-1027

Kozlov M, McMahon H, Chernomordik L (2010) Protein-driven membrane stresses in fusion and fission. Trends Biochem Sci 35(12):699-706

Kummrow M, Helfrich W (1991) Deformation of giant lipid vesicles by electric fields. Phys Rev A 44(12):8356

Manakova K, Yan H, Lowengrub J, Allard J (2016) Cell surface mechanochemistry and the determinants of bleb formation, healing, and travel velocity. Biophys J 110(7):1636-1647

McMahon H, Gallop J (2005) Membrane curvature and mechanisms of dynamic cell membrane remodelling. Nature 438(7068):590-596

Mutz M, Helfrich W (1990) Bending rigidities of some biological model membranes as obtained from the fourier analysis of contour sections. J Phys 51(10):991-1001

Noguchi H, Gompper G (2004) Fluid vesicles with viscous membranes in shear flow. Phys Rev Lett 93(258):102

Noguchi H, Gompper G (2005a) Dynamics of fluid vesicles in shear flow: effect of membrane viscosity and thermal fluctuations. Phys Rev E 72(011):901

Noguchi H, Gompper G (2005b) Shape transitions of fluid vesicles and red blood cells in capillary flows. Proc Natl Acad Sci USA 102(40):14,159-14,164

Okuda S, Inoue Y, Eiraku M, Sasai Y, Adachi T (2013) Reversible network reconnection model for simulating large deformation in dynamic tissue morphogenesis. Biomech Model Mechanobiol 12(4):627-644

Okuda S, Inoue Y, Adachi T (2015) Three-dimensional vertex model for simulating multicellular morphogenesis. Biophys Physicobiol 12:13-20

Peters C, Baars T, Buhler S, Mayer A (2016) Mutual control of membrane fission and fusion proteins. Cell 119(5):667-678 
Powers RJ, Roy S, Atilgan E, Brownell WE, Sun SX, Gillespie PG, Spector AA (2012) Stereocilia membrane deformation: implications for the gating spring and mechanotransduction channel. Biophys J 102(2):201-210

Powers RJ, Kulason S, Atilgan E, Brownell WE, Sun SX, BarrGillespie PG, Spector AA (2014) The local forces acting on the mechanotransduction channel in hair cell stereocilia. Biophys J 106(11):2519-2528

Ramakrishnan N, Kumar P, Ipsen J (2013) Membrane mediated aggregation of curvature inducing nematogens and membrane tubulation. Biophys J 104:1018-1028

Ramakrishnan N, Ipsen J, Kumar P (2015) Organelle morphogenesis by active membrane remodeling. Soft Matter 11:2387-2393

Raucher D, Sheetz M (1990) Membrane expansion increases endocytosis rate during mitosis. J Cell Biol 144(3):497-506
Sato K, Hiraiwa T, Shibata T (2015) Cell chirality induces collective cell migration in epithelial sheets. Phys Rev Lett 115:188102

Schneider M, Jenkins J, Webb W (1984) Thermal fluctuations of large cylindrical phospholipid vesicles. Biophys J 45(5):891-899

Staykova M, Holmes D, Read C, Stone H (2011) Mechanics of surface area regulation in cells examined with confined lipid membranes. Proc Natl Acad Sci USA 108(22):9084-9088

Tsubota K (2014) Short note on the bending models for a membrane in capsule mechanics: comparison between continuum and discrete models. J Comput Phys 277:320-328

van der Ploeg P, Berendsen H (1982) Molecular dynamics simulation of a bilayer membrane. J Chem Phys 76:3271-3276

Zhao S, Kindt J (2005) Monte carlo calculations of the free-energy landscape of vesicle formation and growth. Europhys Lett 69(5):839 